\title{
¿Qué es la traducción financiera? Limitación del concepto mediante la aplicación de diversos parámetros
}

\author{
Elena Alcalde Peñalver \\ Universidad de Granada \\ ealcalde@ugr.es
}

Recibido: 16 de noviembre de 2014

Aceptado: 27 de marzo de 2015

\section{RESUMEN}

El objetivo de este estudio consiste en analizar las características de la traducción financiera para aportar una definición clara de este concepto, lo que contribuiría a facilitar la investigación en las bases para la enseñanza de este tipo de especialidad. Para ello, aplicaremos el modelo de Hatim y Mason (1990) a la traducción financiera para compararla con la traducción económica. Los resultados muestran que, aunque no se puedan establecer unos límites precisos entre los dos tipos de especializaciones, existen ciertas características que definen a la traducción financiera, con las implicaciones que esto tiene para la didáctica y la profesión.

Palabras clave: traducción financiera, traducción económica, discurso y traducción, diseño curricular.

What is financial translation?

Definition of its concept through the application of different parameters

\begin{abstract}
The aim of this study is to analyze the features of financial translation to clarify this concept and help establish the basis of what should be included in a financial translation program. To achieve this, Hatim \& Mason's model (1990) will be applied to financial translation in order to study the features of this type of specialization and compare and contrast it with economic translation. The results show that even though fixed barriers between the two types of specialization cannot be established, there are specific characteristics that define financial translation, which entails implications for the profession and for curriculum development.
\end{abstract}

Keywords: financial translation, economic translation, discourse and translation, curriculum development. 
Sumario: 1. Introducción. 2. Metodología: el modelo de Hatim y Mason. 2.1. La dimensión pragmática aplicada a la traducción financiera. 2.2. La dimensión comunicativa aplicada a la traducción financiera. 2.3. La dimensión semiótica aplicada a la traducción financiera. 3. Hacia una definición propia de traducción financiera. 4. Conclusiones.

\section{Introducción}

Tras observar la aparición de distintos cursos en instituciones de enseñanza pública y privada con la denominación específica de "traducción financiera" o con contenidos de traducción financiera, consideramos que una definición clara de este concepto contribuiría a facilitar la enseñanza de este tipo de especialidad. De esta forma, con el fin de arrojar luz sobre el panorama de la didáctica de la traducción financiera, nos vemos obligados a reflexionar sobre este concepto y definir una serie de criterios que nos permitan acotar los diferentes tipos de contextos en los que enmarcar los textos a los que se enfrentan con regularidad los traductores financieros en el mercado de la traducción.

Las distintas denominaciones de la traducción han servido no solo para estructurar el marco teórico a nivel formativo, sino que también afectan al mercado profesional de la traducción, ya que en muchos casos los honorarios de los traductores se organizan y distinguen de acuerdo con estas categorías. Sin embargo, aunque en el mercado profesional la labor de los traductores financieros sí parece estar delimitada por el ámbito temático en el que se desarrolla, no ocurre igual con asignaturas y otros programas de formación que existen en la actualidad y que incluyen textos procedentes de otras áreas de la economía (Alcalde Peñalver 2014).

Somos conscientes de que autores como Mayoral y Díaz (2011: 19) critican estas clasificaciones tradicionales porque creen que o bien no responden a los principios de cómo debe ser una buena clasificación y resultan poco operativas desde el punto de vista práctico (el cómo traducir) o bien no son el resultado de una profunda reflexión en la naturaleza de la disciplina. Además, consideramos igualmente que no es posible clasificar los estudios de traducción de forma totalmente definida, ya que en numerosas ocasiones los elementos se solapan (Mayoral 2001: 62).

No obstante, no es nuestro objetivo imponer una clasificación de los estudios de traducción, entendiendo clasificación como la organización de elementos en clases diferentes (Mayoral y Díaz 2011: 27), sino simplemente delimitar el concepto de traducción financiera con una finalidad didáctica y rigiéndonos así por el principio de reduccionismo de la categorización clásica (Lakoff 1982: 6). Pretendemos estudiar, a partir de una serie de criterios, si es posible diferenciar la traducción financiera de la económica y categorizarla dentro de lo que se ha denominado tradicionalmente la traducción económica, entendiendo categoría como el procedimiento que lleva a discernir esas mismas clases (Mayoral y Díaz íbid.). 


\section{Metodología: el modelo de Hatim y Mason}

Para analizar si existen diferencias entre la traducción financiera y la traducción económica, adoptamos el modelo de tipología textual de Hatim y Mason (1990), puesto que concebimos la traducción como una actividad compuesta por textos cuyo funcionamiento debe comprender el estudiante, los problemas de traducción que se pueden plantear en ellos y las diferentes estrategias que se pueden adoptar (Hurtado 1996: 38).

Por otro lado, el uso de este modelo viene justificado por el hecho de que consideramos que el traductor realiza una traducción tomando el texto meta como punto de partida, lo que hace que se conciba su trabajo como un proceso textual (Neubert y Shreve 1992: 7). Del mismo modo, Hatim y Mason (1990) y Hatim (2001) defienden de manera general la traducción como un acto comunicativo y textual, además de constituirse como una actividad pragmática que tiene lugar en un contexto social y que cuenta con una serie de fines comunicativos. Coincidimos con los mismos en que la relevancia de los estudios de traducción se basará en que se conciba esta actividad como un acto de comunicación y no como un mero ejercicio lingüístico que no produzca resultados.

Hatim y Mason (1990) analizan el conjunto de procedimientos que posee un texto dentro de su contexto y distinguen en su modelo tres dimensiones que configuran el texto y nos permiten analizar su sentido: la dimensión comunicativa, la dimensión pragmática y la dimensión semiótica. Estas tres dimensiones son interdependientes y se complementan entre sí. A continuación aplicaremos este modelo con sus tres dimensiones al concepto de traducción financiera, lo que nos permitirá analizar la función de los textos económicos y financieros, así como otros elementos como el campo, modo o tono de los mismos. En algunos casos, hemos complementado nuestro análisis con otros modelos para alcanzar un mayor nivel de profundización. El análisis de estos elementos de forma aplicada a la traducción financiera nos permitirá delimitar en mayor medida las características que definen a este tipo de especialidad de traducción.

\subsection{La dimensión pragmática aplicada a la traducción financiera}

La dimensión pragmática hace referencia a la función de los textos. En cuanto a la función de los textos financieros en relación con la de los textos económicos, Ellis y Johnson (1994) señalan que la función de los textos económicos es la informativa y permite el intercambio de información sobre un tema de especialidad. Pizarro (2010: 55) señala que el tipo textual informativo es el más utilizado en los géneros de economía, ya que se emplea para presentar y transmitir la información de forma objetiva, sin intención explícita de convencer.

En el ámbito financiero, coincidimos con Herrero (2011) en que, aunque en él existan muchos textos que parecen informativos, su verdadero propósito es argumentativo, puesto que se busca convencer al receptor, por ejemplo, de las adecuadas condiciones y de la rentabilidad de un producto financiero o del buen estado de una 
determinada empresa para que compre acciones de la misma, tal y como podría ocurrir con un folleto de admisión o unos estados financieros, respectivamente. Sin embargo, los textos económicos tienen un claro objetivo informativo, como realizar un análisis macroeconómico en un informe de coyuntura. Por lo tanto, se podría considerar que mientras que los textos económicos tienen una función meramente informativa, los textos financieros tienen una función argumentativa además de una función secundaria informativa. De esta forma, consideramos los textos financieros como característicos de la naturaleza híbrida que señalaban Hatim y Mason (1990), cuyo foco textual dominante será el argumentativo junto con el informativo de forma secundaria.

\subsection{La dimensión comunicativa aplicada a la traducción financiera}

La dimensión comunicativa se compone de tres elementos que analizamos a continuación: el campo, el modo y el tono de los textos.

En relación al primer elemento de la dimensión comunicativa, consideramos necesario analizar el campo en el que se enmarca la traducción financiera. Para el análisis de esta dimensión hemos decidido adoptar por su exhaustividad y rigor científico la clasificación de la disciplina económica que ofrece la American Economic Association (AEA). Además, no encontramos otra clasificación que para los objetivos de este trabajo nos permitiera establecer unas categorías claras dentro del campo de la economía. Esta asociación favorece la investigación en el campo económico y cuenta con una clasificación por subáreas del mismo para guiar a sus miembros a localizar estudios de esta disciplina de forma más clara y efectiva. Una de las subáreas corresponde a la economía financiera y los temas que incluyen corresponden a los principales elementos de los sistemas financieros, es decir, los mercados, instrumentos e intermediarios financieros.

Esta forma de enmarcar las distintas subáreas del ámbito financiero nos va a servir de punto de partida para distinguir la temática de la traducción financiera y económica. De esta forma, tal y como señalan Hatim y Mason (1990), aunque el campo sea el área temática general en la que se enmarque el texto, las distintas subáreas nos permitirán distinguir los diversos asuntos que se pueden tratar en los textos financieros, como puede ser el caso de documentos sobre la crisis financiera, sobre financiación, fondos de inversión, etc.

En relación al modo, el segundo elemento de la dimensión comunicativa, en el caso que nos atañe nos centramos en la actividad traductora, por lo que este será escrito. No obstante, la comunicación financiera cada vez se produce en mayor medida en el contexto de la interpretación, lo que se observa en las distintas reuniones que se llevan a cabo entre países para tratar temas relacionados con la crisis financiera, o en empresas que trabajan de forma conjunta a nivel internacional, en los que se requiere cada vez con mayor frecuencia servicios de interpretación.

Finalmente, el tono o nivel de formalidad en traducción financiera viene determinado por el tipo de texto que constituye el objeto de traducción. En este sentido, Mateo (2007) destaca el tono coloquial que caracteriza al inglés de la prensa finan- 
ciera. Esto se hace patente en numerosas ocasiones a través de la ironía que se expresa con la metáfora, lo que supone de nuevo un reto para la traducción financiera. Estas metáforas abarcan una amplia gama de áreas semánticas: mecanismos y máquinas, animales (bull market, bear market), plantas y jardinería, salud y forma física, etc. (White 2003). Sin embargo, es necesario subrayar que el mayor número de metáforas lo podemos encontrar en la prensa especializada del ámbito económico, ciertos manuales referidos a este tema y otros recursos de información que encontramos de forma virtual, ya que estos pueden permitirse un mayor grado de coloquialismo.

Del mismo modo, Román (2008) diferencia a su vez el discurso financiero del jurídico y del económico, señalando su carácter coloquial e innovador, que refleja constantemente la realidad de su entorno, mediante el uso, por ejemplo, de expresiones populares y familiares de la vida diaria, como "fondos paraguas" o "goteo". Otros casos de figuras metafóricas que añadiríamos lo constituyen los términos "hora bruja" para referirse a los días de vencimiento trimestral de futuros y opciones, caracterizados por una gran volatilidad del mercado bursátil o haircut, que se puede ver entremezclado en la prensa con su equivalente en español "quita".

Este componente coloquial del discurso financiero también lo señalaron Alcaraz y Hughes (2008) como elemento diferenciador del español de la economía, del que indicaban su registro más elevado y terminología proveniente del francés y el inglés (devaluación, inflación, oligopolio, etc.), mientras que en la lengua de las finanzas el discurso solía ser más informal (fondos paraguas, capital riesgo, goteo) y se adoptaban constantemente nuevos términos de carácter metafórico para dar respuesta a las nuevas realidades de este ámbito.

\subsection{La dimensión semiótica aplicada a la traducción financiera}

La dimensión semiótica se compone de tres elementos que analizamos a continuación: el género, el discurso y el texto.

Para la definición del primer elemento de la dimensión semiótica, vamos a utilizar, al igual que lo hiciera Herrero (2011), el modelo de Gamero (2001: 51), ya que incluye diferentes factores que intervienen en la definición y delimitación de los géneros y que permiten establecer tipologías en el ámbito profesional de la traducción. Este modelo incluirá a su vez características de otros elementos de las dimensiones establecidas por Hatim y Mason, lo que hemos considerado igualmente válido por el carácter interdependiente de las mismas. El modelo de Gamero (2001: 51) hace referencia a los siguientes elementos: los rasgos convencionales de los textos, la función textual, los elementos de la situación comunicativa, el contexto sociocultural y los elementos intratextuales. Estos se explicarán a continuación de forma aplicada a la traducción financiera.

En primer lugar, los rasgos convencionales son aquellos implícitos entre los participantes del proceso comunicativo y que admiten escasa variación. Coincidimos con Herrero (2011) en que, a título ilustrativo, los documentos que engloban los estados financieros o el folleto de admisión a negociación de acciones son géneros 
muy convencionalizados. Más específicamente en el caso de los estados financieros, que constituyen la herramienta más importante con la que cuentan las organizaciones para evaluar el estado en el que se encuentran, estos estarán compuestos, según las NIIF (Normas Internacionales de Información Financiera), por las siguientes partes: balance, cuenta de resultados, memoria, estado de flujos de efectivo y estado de cambios en el patrimonio neto. Las NIIF, a nivel internacional, al igual que el Plan General de Contabilidad (PGC) en el territorio nacional, constituyen una de las principales razones de la convencionalización de los textos financieros, ya que indican los criterios a seguir para la elaboración de ciertos tipos de documentos. Sin embargo, los textos económicos no presentan rasgos convencionalizados que el emisor deba respetar, ya que son meramente informativos, tales como un análisis macroeconómico de un país o el informe mensual de un banco central.

En relación a la función textual, tal y como señalamos anteriormente, Herrero destaca que aunque en el ámbito financiero existen muchos textos que parecen informativos, su verdadero propósito es argumentativo para intentar convencer al receptor, por ejemplo, de las adecuadas condiciones y de la rentabilidad de un producto financiero. Sin embargo, los textos económicos tienen un claro objetivo informativo, como por ejemplo un análisis macroeconómico en un informe de coyuntura.

Entre los elementos de la situación comunicativa encontramos los siguientes: emisor, receptor, campo, tenor y modo. Según Herrero (2011: 94) el campo (actividad profesional de los participantes) y el modo (canal de comunicación y retórica empleada) serían los que mayor grado de distinción permiten mostrar entre el discurso económico y financiero. Coincidimos con la autora en que el campo y modo constituyen factores de diferenciación entre ambos discursos, ya que la actividad de un economista y un experto en finanzas se desarrolla en distintas áreas de profesionalidad. Además, el empleo de recursos lingüísticos del lenguaje cotidiano y de metáforas es más común en los textos financieros, como hemos mencionado previamente. Sin embargo, añadiríamos el emisor y receptor como factores diferenciadores de la situación comunicativa.

En relación al contexto sociocultural, Herrero (2011) señala que entre los cinco parámetros que se utilizan del modelo de Gamero (2001) para la definición del género, este sería el que no establecería ninguna diferencia entre los géneros económicos y financieros. Sin embargo, consideramos que la influencia del ámbito anglosajón, elemento perteneciente al contexto sociocultural, ha resultado en la importación de productos financieros que han dado lugar a necesidades terminológicas que no existían con anterioridad. Este es el caso por ejemplo del conocido capital riesgo o venture capital, que surge a finales de los años cuarenta en Estados Unidos como forma de financiamiento de empresas que no cotizan en bolsa.

Finalmente, en cuanto a los elementos intratextuales, Alcaraz y Hughes (2008: 2) enmarcan el discurso financiero dentro del español de los negocios, diferenciándolo del lenguaje de la economía y el comercio. Según argumentan, el español de la economía es el que menos problemas plantea a los traductores porque, al tratarse de una materia académica, está redactado con un discurso más elevado que los otros dos y su terminología suele coincidir con la francesa y la inglesa (devaluación, inflación, oligopolio, etc.). Sin embargo, afirman que en el lenguaje de las finanzas con- 
fluyen dos factores que dificultan la comprensión de esta variedad y de su posterior traducción: primero, el discurso suele ser más coloquial (fondos paraguas, capitalriesgo, goteo, etc.) y, segundo, constantemente se están acuñando términos para nuevos productos. Además, señalan que en el español de las finanzas se observa una gran dependencia de la terminología inglesa. Finalmente, distinguen dentro del lenguaje de los negocios el lenguaje del comercio que, por la larga tradición de esta actividad, goza de una combinación bastante equilibrada de términos tradicionales y de términos modernos (plancha, estadía, conocimiento de embarque, flete, mercaderías, etc.). No obstante, en un trabajo de investigación previo, Alcaraz señala que esta categorización en tres grandes subáreas la realiza únicamente con fines operativos y por lo tanto siempre tiene en cuenta los fuzzy sets de las mismas, es decir, los bordes o contornos aproximativos que las caracterizan (2001: 12).

Como se ha podido observar, de nuestro análisis se puede concluir que existen también elementos definitorios que confirman la existencia de un género financiero. No obstante, aunque consideremos que existen diferencias entre el género económico y el financiero, no lo concebimos como un compartimento estanco con barreras rígidas en el que no tengan cabida otro tipo de textos, ya que como hemos señalado anteriormente en relación al discurso, habrá ocasiones en las que un texto económico tenga cabida en contextos financieros y viceversa. Sin embargo, no llegamos al punto de coincidir con Heras (2005) en que hoy en día es difícil afirmar de forma contundente que existe un tipo de texto de marcado carácter económicofinanciero. Como hemos podido comprobar sí que existen determinadas características que nos van a ayudar a identificar a un texto financiero, lo que facilitará por lo tanto nuestra labor como traductores y docentes.

Para la delimitación del segundo elemento de la dimensión semiótica, es decir, el discurso, tendremos en cuenta las características de los lenguajes de especialidad señaladas anteriormente por Cabré (1993), que hace referencia a la temática, los usuarios y las situaciones de comunicación.

En primer lugar, en relación a la temática, Herrero (2011) parte de los antecedentes que nos muestran que en los libros o artículos sobre lenguajes especializados, el lenguaje económico y el financiero suele integrarse bajo una misma categoría, ya que de hecho es posible encontrar ambos adjetivos unidos (lenguaje económicofinanciero, traducción económico-financiera).

No obstante, tal y como hemos observado en el apartado dedicado al ámbito temático en el que se puede enmarcar la traducción financiera, ya de por sí este da lugar a que el discurso financiero cumpla con unos fines comunicativos específicos, restringidos al ámbito de las finanzas como subcampo debidamente diferenciado de la economía. Es necesario aclarar que no pretendemos en este caso establecer unos criterios de categorización en función de la temática propia del texto, sino en función de la temática del ámbito en el que se enmarca (Mayoral 2001). Esto se debe a que somos conscientes de que los textos pueden contar con una terminología específica en función del sector de la empresa de la que sean objeto $\mathrm{y}$, por lo tanto, nos encontraremos con ámbitos temáticos específicos según se trate de una empresa de telecomunicaciones, de arquitectura, de productos alimentarios, etc. (Alcalde 2013). 
En este sentido, Herrero (2011) considera que los distintos tipos de textos que se redactan en el ámbito financiero muestran un discurso específico relacionado con las necesidades terminológicas que surgen en este campo. De este modo, establece una serie de categorías en función de las necesidades comunicativas por ámbito temático en el campo financiero y que, aunque nos hayan servido de punto de partida para este análisis, nosotros hemos considerado conveniente adaptar en nuestra investigación en función de la clasificación por área temática del apartado anterior.

Nuestra propuesta de categorización del discurso financiero, siguiendo la clasificación de la AEA y agrupando las peculiaridades señaladas por Herrero en su análisis, sería la siguiente:

Términos sobre la metodología de las finanzas: en esta primera categoría sí coincidiríamos con la última establecida por Herrero, ya que se haría referencia, según menciona la AEA, a "studies about general issues covered in financial economics, including textbooks, survey articles, and data" (AEA).

Términos sobre mercados financieros: en esta categoría se englobarían varias de las establecidas por Herrero. En primer lugar encontraríamos los términos relacionados con operaciones bursátiles (ampliaciones de capital, OPA, OPV, salidas a bolsa). En segundo lugar, distinguiríamos los términos relacionados con los tipos de activos. Entrarían bajo esta categoría, deuda pública (letras del tesoro, bonos y obligaciones del Estado), deuda privada (bonos, pagarés, cédulas hipotecarias, participaciones preferentes), fondos de inversión (fondos monetarios, de renta fija, de renta variable, de gestión pasiva, de retorno absoluto), derivados (futuros, opciones, CFDs). Consideramos que esta última categoría es de gran importancia porque es en el ámbito de los productos financieros donde se produce un mayor grado de innovación.

Términos sobre los intermediarios financieros, instituciones financieras y empresas de servicios financieros. Los intermediarios financieros constituyen un elemento esencial de los sistemas financieros, ya que se encargan de canalizar los ahorros de las unidades con superávit para que lleguen a las unidades deficitarias que necesitan el dinero para la inversión, lo que constituye una característica imprescindible de la economía. Cabe destacar también la importancia de las instituciones financieras encargadas de la regulación de los mercados y servicios financieros (caso por ejemplo de la CNMV) y otras instituciones de servicios financieros como los bancos, agencias crediticias, aseguradoras, etc.

Finalmente, siguiendo la categorización establecida por la AEA y muy en consonancia con la actualidad en materia financiera debido a los numerosos escándalos relacionados con la corrupción y malas prácticas en operaciones financieras, consideramos oportuno añadir una última categoría sobre "financiación empresarial y gobierno corporativo", en la que se englobarían todos los términos relacionados con la gestión sostenible, transparencia, seguridad, etc.

Con esta categorización se pretende mostrar que existe una primera particularidad en el discurso financiero que surge del grado de especificidad del ámbito temático de las finanzas, lo que crea unas necesidades terminológicas determinadas que lo diferencian del lenguaje económico en general. No obstante, no coincidimos con Cabré en que la temática es especializada cuando no forma parte del conocimiento 
general de los hablantes de una lengua, ya que en el ámbito de las finanzas el nivel de familiarización terminológica y comprensión de determinados conceptos por parte del público es cada vez mayor. Esto se debe a la recurrencia con la que aparecen temas financieros en los medios de comunicación y que afectan a los ciudadanos de todas clases de diferentes maneras (temas de las preferentes, hipotecas basura, bolsa, depósitos, tipos de interés, etc.). No obstante, los actos comunicativos del ámbito financiero van a hacer que los textos resultantes sigan siendo considerados como especializados, aunque no de uso exclusivo de especialistas, tal y como se argumentará en el siguiente apartado.

Por otro lado, en relación a los usuarios del discurso financiero, consideramos que existen agentes específicos en la comunicación financiera que establecen su campo de acción en este ámbito (López 2003: 47-48):

En primer lugar, entre los emisores podemos destacar los emisores primarios de valores y otros productos financieros, entidades crediticias, aseguradoras, empresas de servicios de inversión, formadores y líderes de opinión, organismos de control.

Entre los receptores encontramos los ahorradores, inversores institucionales, formadores y líderes de opinión, empresas de servicios de inversión, organismos de control.

Sin embargo, no coincidimos con Hurtado (2001) en el hecho de que los usuarios sean especialistas. Como hemos observado previamente, aunque la mayoría de los emisores de los documentos financieros sean expertos (entidades crediticias, empresas de servicios de inversión, etc.), entre sus usuarios se encuentran igualmente ciudadanos de a pie, puesto que el grado de información financiera con el que cuentan es cada vez mayor.

En relación al concepto de situación comunicativa, tal y como señalábamos en el apartado anterior, en traducción financiera va a venir determinada por un emisor especialista que emitirá documentos con el objetivo de hacer llegar información a una serie de receptores, ya sean especialistas o no especialistas. El concepto de situación comunicativa de experto a experto está cambiando en la actualidad por el mayor grado de información financiera al que tiene acceso el público general, tal y como señalamos anteriormente. Por lo tanto, se amplía el abanico de personas que entran a formar parte de las situaciones de comunicación en las que se pueden producir casos de traducción financiera. De este modo, consideramos que existirán tantas situaciones comunicativas como necesidades de tratar asuntos determinados del campo de las finanzas se puedan producir.

Finalmente, en relación al tercer y último elemento de la dimensión semiótica, consideramos de gran utilidad la definición de los principales tipos de textos que forman parte del género financiero, ya que esto permite aportar una visión panorámica de lo que se traduce para posibilitar la comunicación financiera. Estos textos contarán con las características anteriormente enunciadas, aunque esto no quiera decir que todos ellos se ciñan a los mismos criterios y se muestren caracterizados en su totalidad de forma homogénea. Consideramos que, partiendo del análisis del ámbito temático que mostramos anteriormente, podríamos extraer de forma más clara para el lector los principales textos que son objeto de distintas situaciones comunicativas en función del ámbito en el que se enmarcan. 
A continuación agruparemos los principales textos que nos encontramos en el ámbito financiero teniendo en cuenta la situación comunicativa del área temática en la que se enmarca. Sin embargo, al no ser el objetivo de este estudio analizar en profundidad este tipo de textos, nos limitaremos a enumerarlos, definirlos brevemente y acompañarlos de una explicación acerca de su utilidad en el campo financiero. Para ello, de nuevo nos basaremos en la categorización establecida por la AEA. Es necesario señalar que en ningún momento pretendemos que esta categorización sea exhaustiva ni extrapolable a todos los estudios de traducción financiera. Se recogen los textos más representativos del mercado de la traducción en el ámbito financiero según nuestra experiencia, las consultas realizadas a profesionales y la literatura existente. No obstante, para profundizar en otras propuestas clasificatorias de textos financieros y la terminología propia de los mismos se puede consultar el trabajo de Román y Herrero (2014) y los resultados del proyecto COMENEGO (Corpus Multilingüe de Economía y Negocios) de la Universidad de Alicante, que a lo largo del 2013 realizó una encuesta a prestadores de servicios de traducción para conocer los principales tipos de textos de este ámbito que suelen traducir.

En primer lugar, en relación con la categoría sobre la metodología de las finanzas, podríamos distinguir entre textos financieros presentes en manuales, libros de divulgación y artículos periodísticos. En este caso, su utilidad se basa en la aportación de información al lector sobre distintos asuntos financieros para que pueda conocerlos de forma más detallada.

En segundo lugar, dentro de la categoría de mercados financieros podríamos distinguir los siguientes textos: folletos de emisión, informes de brókers, notas de prensa, estados financieros y códigos de buen gobierno. Estos textos están caracterizados por contener terminología propia del lenguaje especializado de las finanzas, puesto que se producen en este campo temático con objeto de tratar información sobre distintos asuntos de especialidad, responden al género de las finanzas y dan respuesta a situaciones de comunicación determinadas.

\section{Hacia una definición propia de traducción financiera}

En primer lugar, hay que señalar que tras el análisis realizado hasta este punto del trabajo, enmarcamos la traducción financiera dentro de lo que Hurtado (2001:59) denomina "traducción de textos especializados" y no "traducción especializada". Según la autora este último concepto abarcaría toda la traducción de textos, ya que para ello se requieren unos conocimientos y habilidades especiales. Del mismo modo, tal y como hemos mencionado con anterioridad, tendremos también en cuenta la definición de Cabré (1993: 139) según la cual los lenguajes de especialidad "son subconjuntos del lenguaje general caracterizados pragmáticamente por tres variables: la temática, los usuarios y las situaciones de comunicación".

En función de las características analizadas en los apartados anteriores, consideramos que los tres criterios fundamentales sobre los que se debe articular la definición del concepto de traducción financiera son los siguientes: temática, discurso y género, que entrarían dentro de la dimensión semiótica del modelo de Hatim y Mason. 
En relación a la temática, podemos señalar que consideramos las finanzas como un subcampo de la economía en general. El discurso financiero se caracteriza por el dinamismo terminológico debido a la constante innovación y rápida evolución en este ámbito, lo que hará que exista una cierta influencia anglosajona y se adopten términos directamente del inglés. Del mismo modo, el discurso financiero está marcado por el uso de figuras retóricas como las metáforas, lo que le aporta un cierto grado de coloquialismo y las asimetrías culturales son prácticamente inexistentes. Finalmente, en relación al género, los textos financieros muestran un alto nivel de convencionalización, con una función argumentativa predominante y una informativa secundaria, cuyos emisores serán expertos (entidades financieras, organismos de control) y los receptores podrán ser tanto expertos como el público general que tenga interés personal en adquirir algún tipo de información relacionada con sus finanzas personales.

De esta forma, agrupando un mayor número de características, consideramos que una primera definición más extensa del concepto de traducción financiera podría ser la siguiente:

La traducción financiera es una rama de la traducción que da cuenta de las necesidades comunicativas que emanan del campo de las finanzas. Se trata de una especialidad muy amplia dentro del campo de la traducción económica y que está dotada de rasgos singulares que la distinguen de otras parcelas dentro de este tipo de traducción. Entre estas características encontramos un discurso marcado por el dinamismo terminológico debido a la constante innovación y rápida evolución de este ámbito, lo que provocará a su vez que exista una cierta influencia anglosajona y se adopten términos directamente del inglés. Del mismo modo, existe un alto uso de figuras retóricas como las metáforas, lo que le aporta un cierto grado de coloquialismo y las asimetrías culturales son prácticamente inexistentes. Además, los textos financieros son altamente convencionalizados, con una función principalmente argumentativa y una secundaria informativa, cuyos emisores serán expertos (entidades financieras, organismos de control) y los receptores podrán ser tanto expertos como el público general que tenga interés personal en conocer algún tipo de información relacionada con sus finanzas personales.

No obstante, consideramos que una definición que abarca las características principales, sin entrar en tanto detalle, podría ser la siguiente:

Actividad que conlleva la traducción de textos especializados dentro del área de las finanzas, entendiéndola como un subcampo de la economía en general y cuyos textos presentan un alto grado de convencionalización, una función principalmente argumentativa y una secundaria informativa y un discurso que vendrá marcado por la innovación terminológica característica de este ámbito, con la consiguiente aceptación de un gran número de anglicismos, al igual que por un cierto grado de coloquialismo, debido al uso de figuras retóricas como la metáfora.

Esta definición, tanto en su versión amplia como resumida, constituye una forma de sintetizar todo el estudio realizado sobre el concepto de traducción financiera. En ella intentamos reconocer el gran número de elementos que se pueden incluir en el concepto. En ningún momento pretendemos defender esta definición como única, puesto que somos conscientes de la dificultad de aunar todas las peculiaridades que 
ya de por sí tienen los campos de la traducción y las finanzas como campo de especialidad. Por lo tanto, tal y como señala Tymoczko (2005: 1082) en relación al concepto de traducción, la traducción financiera puede también considerarse un cluster conceptual, es decir, un concepto conglomerado con una naturaleza definitoria abierta. Sin embargo, esperamos que la limitación del ámbito temático con los diferentes tipos de textos que mencionamos como más representativos y que están caracterizados de forma general por los criterios de discurso y género analizados permitan, como dijimos anteriormente, dotar de mayor claridad a las bases curriculares en traducción financiera y que el traductor profesional sepa enmarcar el texto objeto de traducción con mayor facilidad en su área temática y hacer que el proceso de documentación y búsqueda terminológica se vuelva más efectivo.

\section{Conclusiones}

Hemos procedido a la delimitación del concepto de traducción financiera mediante la aplicación del modelo teórico de Hatim y Mason que contempla tres dimensiones: la pragmática, la comunicativa y la semiótica. Aunque, como se ha podido observar, las tres dimensiones hayan contribuido a la delimitación del concepto, consideramos que la de mayor utilidad ha sido la semiótica, ya que nos ha permitido distinguir los tres conceptos básicos para acotar nuestro concepto de traducción financiera: género, discurso y texto. Por ello, nos hemos servido de estos tres elementos para aportar una definición final de lo que se podría considerar la traducción financiera. Esta definición constituye una simple aproximación que reúne las características principales de esta especialidad de traducción analizadas en este trabajo. Es necesario indicar que ha sido necesario complementar el modelo de Hatim y Mason con los criterios delimitadores aportados por otras investigaciones como la de Cabré (1993) para acotar el discurso financiero, o el modelo de Gamero (2001) para el concepto de género financiero. Esto nos ha permito analizar el concepto de traducción financiera con un mayor grado de profundidad y estudiarlo desde puntos de vista que complementaban el modelo teórico de referencia aplicado.

\section{Referencias bibliográficas}

Alcalde Peñalver, E. «La traducción del balance de situación: análisis de dificultades y propuesta de actividades mediante un estudio de corpus». En Ortega, E. (Dir.) Translating Culture. Granada: Comares 2013, 437-448.

Alcalde PeÑalver, E., Caracterización y contextualización de la traducción financiera: estudio empírico-descriptivo de la situación académica y profesional en España. Tesis doctoral. Universidad de Granada 2014.

Alcaraz Varó E. y Hughes, B., Diccionario de términos económicos, financieros y comerciales: inglés-español. Barcelona: Ariel 2008.

AlCARAZ VARÓ, E. «Problemas metodológicos de la traducción del inglés de los negocios», en: Proceedings. First International Conference on Specialized Translation. Barcelona: Universitat Pompeu Fabra 2001, 11-21. 
AMERICAN ECONOMIC CLASSIFICATION. Journal of Economic Literature (JEL) Classification System. Disponible en http://www.aeaweb.org/jel/jel_class_system.php [Último acceso 06/06/14].

CABré Castellví, M. T., La terminología: teoría, metodología, aplicaciones. Barcelona: Antártida/Empúries 1993.

Comenego, Encuesta a prestadores de servicios de traducción. Disponible en http://dti.ua.es/es/comenego/encuesta-a-prestadores-de-servicios-de-traduccion.html 2013 [Último acceso 6/04/13].

Ellis, M. y Johnson, C., Teaching Business English. Oxford: Oxford University Press 1994.

Gamero Pérez, S., La traducción de textos técnicos: Descripción y análisis de textos (alemán-español). Barcelona: Ariel 2001.

Hatim, B. y Mason, I., Discourse and the translator. Londres: Longman 1990.

Hatim, B., Teaching and Researching Translation. Essex: Pearson 2001.

HERAS DIEZ, F. Materiales para la traducción económica-financiera francés-español. Alicante: Club Universitario 2005.

HERrero, L., «La conveniencia de una clasificación de los documentos del ámbito financiero para la traducción especializada», Entreculturas, 3 (2011), 85-98.

HurTADO AlBIR, A., «La traductología: lingüística y traductología», TRANS. Revista de Traductología. Universidad de Málaga 1996.

Hurtado Albir, A., Traducción y Traductología. Introducción a la Traductología. Madrid: Cátedra 2001.

Lakoff, G., Categories and Cognitive Models. Indiana University Linguistics Club 1982.

LÓPEZ HitA, R. Introducción a la comunicación financiera. Castelló de la Plana: Publicacions de la Universitat Jaume I 2003.

Mateo Martínez, J., «El lenguaje de las Ciencias Económicas». En Alcaráz, E., Mateo, J. y Yus, F. (Eds.) Las lenguas profesionales y académicas. 2007, 191-203.

Mayoral Asensio, R. y DíAz Fouces, O., La traducción especializada y las especialidades de la traducción. Castelló de la Plana: Universitat Jaume I 2011.

Mayoral Asensio, R., Aspectos epistemológicos de la traducción. Castelló de la Plana: Publicaciones de la Universitat Jaume I 2001.

Neubert, A. y Shreve, G. M., Translation as Text. Kent: The Kent State U.P 1992.

Pizarro SÁnchez, I., Análisis y traducción del texto económico. La Coruña: Netbiblio 2010.

Román Mínguez, V. y Herrero, L. «La traducción inglés-español de los géneros de la economía y las finanzas». Comunicación presentada en el Congreso Internacional de Traducción Económica, Comercial, Financiera e Institucional organizado en la Universidad de Alicante 2014.

RomÁn Mínguez, V., La enseñanza de la traducción (inglés-español) de los tipos textuales del mundo de la empresa: aproximación metodológica y materiales. Tesis doctoral. Málaga: Universidad de Málaga 2008.

TymoczKo, M. «Trajectories of Research in Translation Studies». Meta, 50, 4 (2005), 10821097.

White, M., «Metaphor and economics: The case of growth». En English for Specific Purposes, 17, 1 (2003), 3-14. 